\title{
The Importance of Schmidt Astrometry for the Optical Identification of Sources detected in other Wavebands
}

\author{
Ann Savage \\ UK Schmidt Telescope, Anglo-Australian Observatory, Coonabarabran, \\ NSW 2357
}

Russell D. Cannon

Anglo-Australian Observatory, PO Box 296, Epping, NSW 2121

\begin{abstract}
The early optical identifications of radio sources were restricted to bright galaxies and to quasars with ultraviolet excesses, since such objects were rare and unlikely to land by chance within the large radio position error boxes. Other types of objects, such as very high redshift quasars and distant galaxies, were discriminated against because they did not look unusual and so were not readily recognised. Eventually much more accurate interferometric radio positions became available, enabling unambiguous identification of many optically faint objects. Now the internal accuracy of the radio positions can exceed that of the optical positions, requiring optical astrometry to be done to higher precision than ever before. A related problem is the correct registration of the two reference frames, which should be solved soon using a combination of data from the Hipparcos satellite and the Hubble Space Telescope.

The digitised data now available from the Schmidt sky surveys make it possible to automate the optical identification of sources, such as those in the IRAS infrared and Rosat X-ray surveys. Very accurate digitised optical surveys are also needed to prepare lists of targets for multi-object spectroscopy using fibre optic systems.
\end{abstract}

\section{Introduction}

The first great development of non-optical astronomy was at radio wavelengths and much of this paper is concerned with the history of the identification of radio sources. This provides many examples of the difficulties and pitfalls in making secure cross-identifications; similar paths are being followed in X-ray and infrared astronomy. Current work focuses on the accurate registration of the radio and optical reference frames, so that secure identifications can be made on the basis of positional coincidence alone. Recently, machine-generated optical databases have become available covering the whole sky and these are starting to be used for the wholesale identification of sources. One particular aspect of this work is the preparation of input positions for modern multi-object spectroscopic systems using optical fibres. Since the fibres typically have diameters of about two arcsec, the relative positions have to have an r.m.s. accuracy of 
about 0.2 arcsec. In what follows, many of the examples come from the use of the UK Schmidt Telescope for the identification of Parkes radio sources, since that is the field with which the authors are most familiar. However, we believe that the techniques are more generally applicable and will become increasingly important as the positional precision at other wavelengths improves and as the search for exceptional objects continues.

\section{The Identification of Radio Sources}

\subsection{A brief historical review}

The history of radio astronomy illustrates the importance of accurate positions for obtaining reliable optical identifications of sources. When radio astronomy was in its infancy it was naturally assumed that discrete sources must be stars of some sort. John Bolton and his collaborators (Bolton, Stanley \& Slee 1949) were the first to make reasonably accurate positional measurements for three of the strongest radio sources, using their 'sea interferometer' mounted on a cliff top near Bondi Beach in Sydney and a similar instrument in New Zealand. There were no bright stars in the positions corresponding to the sources but, having recourse to the famous astronomical database Norton's Star Atlas, they found that the very unusual galaxy NGC 5128 coincided with the position of Centaurus $A$, the brightest radio source in that constellation. The other two sources turned out to be the Crab Nebula, a supernova remnant, and the elliptical galaxy M87. The still amazing result of this was the realisation that the radio view of the universe is very different from the optical picture. In fact very few stars are easily detectable as radio sources, which is at the root of the problem of tying together the radio and optical reference frames.

The positions which Bolton et al. (1949) used were accurate to about $10 \mathrm{arcmin}$. Quite a few other radio sources were identified with galaxies over the following decade but the next breakthrough required much higher positional accuracy. By combining two large antennas at Owens Valley as an interferometer it was possible to obtain positions accurate to 5 arcsec. Using these, Matthews and Sandage (1963) found faint (16-17 mag) stellar objects at the positions of $3 \mathrm{C} 48,3 \mathrm{C} 196$ and $3 \mathrm{C} 286$, but were initially unable to interpret the very unusual spectra of these 'radio stars'. Hazard, Mackey and Shimmins (1963) then used the technique of lunar occultations at Parkes to fix the position of $3 \mathrm{C} 273$ to better than 1 arcsec. Schmidt (1963) obtained a spectrum at Palomar and recognised that the strong emission features in the thirteenth magnitude 'star' at the position included the Balmer series of hydrogen, but very much redshifted. Quasars had been discovered.

The existence of radio galaxies and quasars was completely unexpected and both discoveries could only be made when radio positions became sufficiently accurate to make unambiguous identifications with the corresponding optical images. However, once the types of objects had been recognised it was possible to make many more identifications from less accurate radio positions. Although the positions in the Parkes Catalogue of Radio Sources (Bolton, Gardner \& Mackey 1964) are accurate to only about 0.6 arcmin, identifications could be suggested for bright galaxies or for quasars, as evidenced by their ultraviolet excess (Bolton, Clarke \& Ekers 1965), because both these types of objects are 
relatively rare. There are only about $0.6 \mathrm{UV}$ excess objects and 7.7 galaxies per square degree to $B$ magnitude 17.5. Identifications with galaxies could be made from the Palomar Observatory - National Geographical Society Sky Survey (POSS) carried out a decade or so previously with the $1.2 \mathrm{~m}$ Schmidt Telescope (now the Oschin Schmidt Telescope). It turned out that it was also possible to recognise quasars on pairs of blue and red Schmidt photographs by what appeared to be a 'blue excess', although that was probably mainly due to the out-of-focus UV light (Bolton, private communication).

There are however two obvious drawbacks to identifying radio sources in this way, based on relatively poor positions. First, new rare classes of objects will not be recognised; you only find what you are looking for. Second, false identifications may be claimed. The subsequent history of quasars illustrates both points. It took many years to make much progress with finding high redshift quasars, simply because most quasars with redshifts greater than 2.7 do not have UV excesses. And it soon became apparent that there were many more UV excess stellar objects than there were radio sources, leading to Sandage's (1965) announcement of radio quiet QSOs as 'a major new constituent of the universe'. Radio interferometry began producing precise positions for large samples of strong sources in the late $1960 \mathrm{~s}$, allowing the identification of radio galaxies at the limit of the POSS by positional coincidence alone.

\subsection{The cautionary tale of PKS $2000-330$}

Murdoch (1993) gives an entertaining account of the importance of accurate positions in the quasar story, to eliminate redshift biases and to aid the recognition of objects already catalogued, while Hunstead (1994) reviews the history of radio galaxy identifications. Both authors give examples of the pitfalls in this work. The history of PKS 2000-330 is illuminating. The Tidbinbilla Interferometer was used to obtain arcsecond positions (Jauncey et al. 1982) for all the Parkes $2.7 \mathrm{GHz}$ Survey flat spectrum sources south of $-30^{\circ}$ declination. A finding chart for PKS 2000 - 330 is shown in Fig. 1. The original Parkes position is indicated by the larger circle; object $A$ near the centre has a UV excess and was assumed to be the identification. However, the much better Tidbinbilla position, indicated by the smaller circle, is right on the edge of the earlier error circle. It eliminates object $A$ and coincides with an apparently uninteresting red star. Spectroscopy with the Anglo-Australian Telescope revealed that this was in fact a quasar at the then record redshift of 3.78 (Peterson et al. 1982). It had been 10 years since the discovery of the previous highest redshift quasar and PKS 2000-330 was to remain the most distant radio quasar for the next 10 years. The ability to identify such radio sources on the basis of positional coincidence alone, without recourse to colour or morphology, is crucial. Identification criteria such as ultraviolet excess and morphology have in the past created serious biases in the resulting redshift distributions.

\subsection{Radio positions surpass optical astrometry}

While early southern radio source identification work was limited by the accuracy of the radio positions, surveys such as that with the Tidbinbilla Interferometer (Jauncey et al. 1982) gave radio positions with an accuracy of 1-3 arcsec and highlighted the need for an improved optical astrometric grid. Residuals from the 


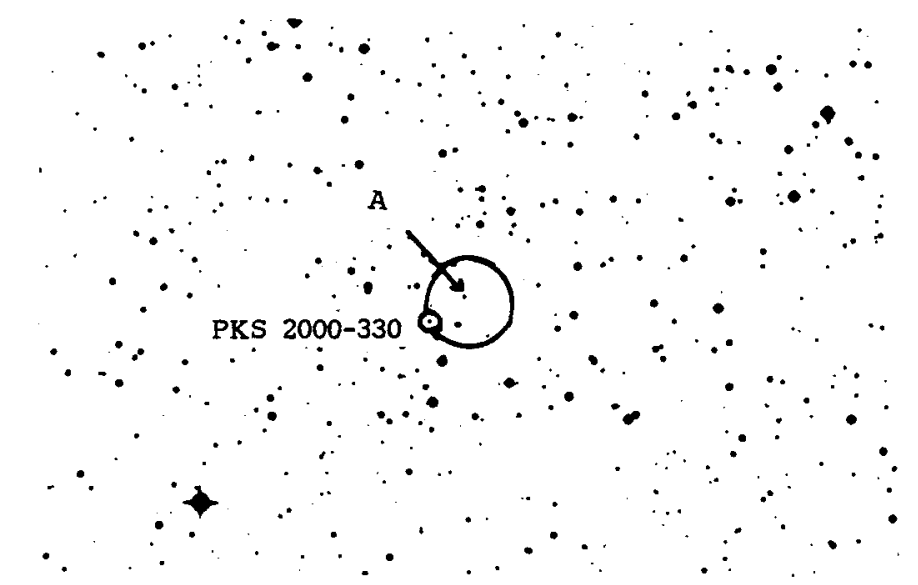

Figure 1. A finding chart for PKS $2000-330$

reference star positions, as measured on Schmidt sky survey photographs, were found to increase dramatically with increasing southern declination (e.g. from $0.85 \operatorname{arcsec}$ near the equator to $2.0 \mathrm{arcsec}$ at $-80^{\circ}$ ) and also to increase with time, i.e. as more recent plates were used, indicating that incorrect proper motions were being applied. Thus the optical position errors were now dominating and defining the error circle radius. Since then, the radio positions have continued to improve as ever longer baselines have been used for interferometry, further highlighting the shortcomings of the southern optical catalogues.

Until recently, the SAO Star Catalogue was used for most radio source identification work. This catalogue conveniently covers the whole sky. However, it is a compilation from several earlier catalogues and is not done to uniform accuracy or on a single astrometric system. In particular, the data for the southern hemisphere are significantly worse than for the north. This situation is now rapidly improving. The PPM catalogue (Röser \& Bastien 1988) now covers the southern sky as well as the northern, with r.m.s. deviations typically at the 0.5 arcsec level. Once the Hipparcos astrometric satellite data are released (due in 1996), there will be a single very accurate and self-consistent optical reference frame covering the whole sky.

Apart from local errors in the optical reference system, there is the problem of large scale systematic errors. Early on, the radio positional system was tied to the optical system using previous identifications; as more sources were identified, the radio positional grid was progressively improved through an iterative procedure. However, to this day relatively few radio sources have been identified south of $-45^{\circ}$. The problems which this can cause are illustrated by the case of the recent supernova, SN1987A. The beautiful optical images taken with the HST, showing an elliptical ring about one arcsecond in diameter surrounding the resolved central supernova remnant, are well-known. After a brief radio outburst at the time of the explosion in 1987, the supernova became undetectable for several years. However, since mid-1990 it has been slowly increasing in radio luminosity. The question is: where is the radio emission coming from? The expanding central supernova remnant seems the most likely candidate, but initially 
the best radio and optical positions were offset in position by about 0.5 arcsec, indicating that the radio emission was coming from a point nearer the HST ring (Staveley-Smith et al. 1992). Fortunately this dilemma has been resolved with the aid of Hipparcos and the best current map does show the supernova located at the centre of the radio emission (Reynolds et al., private communication).

\subsection{Linking the radio and optical reference frames}

To link the radio and optical frames it is necessary to have point sources which can be detected in both frequency ranges. Johnston (1994) reviews the present status of this work. Quasars with core radio emission are ideal candidates, with the added advantage of having no discernible proper motions. A VLBI programme of positioning compact sources to subarcsecond accuracy has led to the identification of many new southern sources, including some suitable as reference sources (Jauncey et al. 1989).

An accurate inertial radio reference frame with milliarcsecond accuracy can be defined by observations of quasars. Jauncey (1989) and Reynolds (1994) have given reviews of VLBI astrometry and its use in linking the radio and optical reference frames. The launch of the Japanese VLBI satellite VSOP and the Russian 'Radioastron' will provide the opportunity to image radio sources over the whole sky, to give milliarcsecond radio positions. However, the optical counterparts will be too faint to be included in the optical frame defined by Hipparcos, which is tied to local stars and hence includes Galactic rotation. One solution to this problem is to use the fine guidance sensors of the Hubble Space Telescope to determine the angular separations between Hipparcos stars and the optical images of quasars, using the HST's capability to measure precise angular distances between objects of disparate magnitudes. This will achieve the simultaneous goals of tying the optical and radio systems together and linking both to an inertial extragalactic system.

Are there more bright quasars to be found in the southern hemisphere? The Einstein, IRAS, ROSAT and Edinburgh-Cape surveys all discovered some and may produce more. For example, IRAS led to the discovery of a small number of QSOs brighter than 16.0 magnitude, in both hemispheres, which had not been discovered by previous surveys. There were claims that these were a new type of QSO, but Clowes, Leggett and Savage (1991) disagreed and concluded that their discovery merely reflects the incompleteness of the northern PBQS (Schmidt \& Green, 1983) and the lack of a similar survey in the south. This conclusion is supported by inspection of the Hewitt and Burbidge (1987) compendium of QSOs, which contains 188 objects with $\mathrm{B} \leq 16.5$. Of these, $76 \%$ are in the northern hemisphere and only $24 \%$ in the southern, and with only $12 \%$ lying outside the area of the PBQS survey. Although the radio survey best suited to finding southern quasars is the Parkes $2700 \mathrm{MHz}$ survey (Bolton et al. 1979 and references therein) because it covers all the southern sky, the high survey frequency and flux limit determine that most of the quasars are optically faint (around magnitude 19) and by this magnitude the ratio of radio quiet QSOs to quasars has risen to about 100:1. A radio study of the PBQS sample (Kellermann et al. 1989) suggests that only about 15 to $20 \%$ of the optically bright QSOs will also be detected as quasars in the Parkes radio survey, but many of the remaining bright QSOs will exhibit some radio emission. 
The Edinburgh-Cape survey for UV bright objects is described elsewhere in these proceedings (Stobie et al. 1995). In another current survey described here, Sealey et al. (1995) are using short exposure objective prism plates from the UK Schmidt Telescope to find relatively bright QSOs. The conclusion from all this is that a substantial number of bright QSOs remain to be found in the southern hemisphere, some of which will have sufficient radio luminosity to be useful as optical-radio position calibrators. We need to discover these objects.

\subsection{Biased searches for radio source identifications}

So far we have considered the identification of radio sources from existing surveys, showing the importance of having accurate radio and optical positions to find the rarer classes of objects and to avoid making mis-identifications. However, if the objective is to find specific types of rare object then it may be more efficient to use additional selection criteria before moving to the optical identification stage, particularly if the optical objects are very faint.

Several authors (e.g. Peterson et al. 1982) have noted that high redshift quasars tend to have a peak in their radio spectra at $1 \mathrm{GHz}$. O'Dea (1990) has re-examined this question with the larger sample of quasars now available in the Hewitt and Burbidge (1987) catalogue. Of the 21 high redshift $(z>3)$ quasars, 14 have sufficient data that the radio spectrum can be determined and half of these appear to be gigahertz-peaked sources. Changes of radio spectral shape also occur for high redshift radio galaxies. Blumenthal and Miley (1979) reported that the sources with the steepest radio spectra have the smaller angular sizes, the largest redshifts and the highest $178 \mathrm{MHz}$ monochromatic luminosities. McCarthy (1993) reviews the current situation for high redshift radio galaxies and speculates on how one might design a survey to give the greatest yield of $z>2.0$ galaxies. Such a search should probably concentrate on reasonably strong radio sources that are not detectable on Schmidt sky surveys, which puts them somewhat beyond the scope of this paper. However, McCarthy goes on to warn that 'the most clever selection criteria will probably produce only modest gains over the more easily understood unbiased surveys'.

\section{X-Ray Surveys}

\subsection{The Einstein EMSS survey}

The identification of X-ray sources has followed a similar path to that described above for radio sources. As an example, we can consider the Einstein Observatory Extended Medium Sensitivity Survey (EMSS). There are $835 \mathrm{X}$-ray sources in the EMSS, a sample of faint X-ray sources discovered serendipitously in fields centred on targets at high galactic latitude (Gioia et al. 1990). The goal of the survey was to provide an inventory of the discrete sources which make up the faint X-ray sky. The EMSS X-ray source positions have an accuracy of 35 to 70 arcsec, which makes the determination of the correct optical counterpart a nontrivial task, even at high galactic latitudes where few candidates are visible on the POSS. Stocke et al. (1991) describe the two- step procedure used to identify the sources. The first step determines if the proposed optical counterpart is plausible, based upon optical spectroscopy and X-ray-to-optical flux 
ratio. Optical spectra were obtained for objects within each error circle until the first plausible counterpart was located. The second step of the identification process is to validate the proposed optical classification of the counterpart, using further spectroscopic and imaging criteria. Most EMSS sources were identified with previously known classes of X-ray emitters, apart from five sources identified with cooling flow galaxies with novel attributes. Also, almost all the EMSS AGN are spectroscopically similar to AGN found by other means, although a few resemble normal galaxies so closely that they would not easily be identified as AGN by any other method.

Despite the care which was taken, the EMSS identification procedure can be criticised because it depends upon the properties of previously identified sources to determine plausible optical counterparts. Thus, although the use of a discriminant facilitates the identification procedure for sources with large position errors, it also adds unknown biases and is liable to lead to classes of objects being overlooked.

\subsection{An example from the ROSAT survey}

As a further example of the importance of accurate positions, we consider the identification using ROSAT of new BL Lac objects which could have been discovered earlier as radio sources. Fleming et al. (1993) showed that three objects in the Palomar-Green survey (Green et al. 1986), which were previously classified as DC white dwarfs, are in fact BL Lac objects. Unfortunately, two of these objects had incorrect positions in the PG catalogue; both coincide with previously catalogued radio sources. The third had been found independently as a radio source but the cross-identification with the PG catalogue had been missed.

\section{Identifications using digitised databases}

\subsection{Modern radio source identifications}

The identification process can now be done automatically, by correlating digitised Schmidt plate data with other catalogues of objects. A detailed study of the automated optical identification of radio sources has been made by Unewisse et al. (1993). Although $x, y$ positions on the plate are measured by COSMOS to better than 2-3 $\mu \mathrm{m}(<0.2$ arcsec), errors in the transformation from the plate frame $(\mathrm{x}, \mathrm{y})$ to the frame of the sky $(\alpha, \delta)$ can result in a final positional uncertainty of 1-2 arcsec. Further, internal positional errors due to emulsion or plate distortions, optical distortions in the corrector lens and filters of the telescope, atmospheric refraction and other effects, can lead to an increase in the positional uncertainty if a global solution is applied over the entire plate, although this is generally not a problem locally if optical positions can be calibrated by enough reference stars in small regions of the plate. Taff et al. (1990) found large scale 'swirling' patterns in the residuals from measurements of Schmidt plates for the HST Guide Star Catalogue. Irwin (1994) finds similar results for plates from the Palomar (Oschin) Schmidt but a much smaller effect for the UK Schmidt Telescope. His conclusion is that the pattern is due to the failure of the plate to conform to the spherical focal surface, almost certainly due to errors in 
the plateholder shape. Since the patterns are repeatable they can be corrected through a look-up table procedure, once they have been determined.

A programme is under way (Tasker et al. 1993) to identify sources in the new Parkes-MIT- NRAO (PMN) 4.85GHz radio survey of the southern sky (Griffith \& Wright 1993). The positional accuracy is $\sim 6$ arcsec for strong sources and degrades to $\sim 30$ arcsec for weak sources. A programme to improve the precision of some 8000 PMN source positions, to an accuracy of the order of one arcsecond, is nearing completion using the Australia Telescope Compact Array. The next step is to make optical identifications using the SERC J sky survey material as digitised by COSMOS, originally prepared for the ROSAT identifications (Yentis et al. 1992). For an initial batch of 645 sources an identification level of $30 \%$ has been attained, with a chance coincidence rate of less than $5 \%$ at the $98 \%$ completeness level, using positional coincidence alone (Jackson, private communication). The radio positional accuracies obtained here and the identification rate of $30 \%$ confirm that the extragalactic radio-optical reference frame in the southern hemisphere has improved. A great deal of effort has gone into improving this reference frame (White et al. 1991, and references therein) although there are still radio-optical differences of 1.5 arcsec at the one sigma level.

\subsection{The identification of IRAS sources}

Wolstencroft and collaborators (Wolstencroft et al. 1986; Savage et al. 1987) used an automated procedure to identify the optical counterparts of IRAS point sources, covering 3000 square degrees around the South Galactic Pole. The positions and magnitudes were obtained using UK Schmidt Telescope plates scanned by COSMOS. As well as the problems described by Unewisse et al. (1993), a further difficulty was caused by the large positional errors associated with the IRAS positions, giving a high probability of a chance coincidence with an unrelated object. Fortunately, most IRAS identifications appear to be with rare bright objects, i.e. stars brighter than 11.0 magnitude or galaxies brighter than 17.0 magnitude.

Sutherland et al. (1991) and Wolstencroft et al. (1992) have extended this identification programme to the IRAS Faint Source Catalogue (Moshir et al. 1989), although the chance coincidence rate is very much higher. Wolstencroft et al. used a 'likelihood ratio' criterion to determine the authenticity of candidate identifications and noted that setting the criterion to give a high level of completeness implies low reliability. Sutherland and Saunders (1992) discuss the general use of the likelihood ratio technique for source identification and derive the probability that any candidate is the correct identification. They conclude by emphasizing that this method should be treated as an adjunct to spectroscopic identifications rather than a substitute. Using the reliability estimates they present, it should be possible to optimise the efficiency of making such identifications with the minimum wastage of scarce telescope time.

\subsection{ROSAT identifications}

The ROSAT all-sky survey sources are also being identified using digitised optical plate material (Voges 1992) and again the X-ray source position errors arf very similar to those of the early radio surveys. Many of the X-ray sources are 
bright stars which eases the identification problem, but still about $25 \%$ of the claimed identifications are probably coincidental. In this case, Schmidt objective prism plates are being used as an extra discriminant. The objective prism spectra were found to be especially useful in identifying $M$ stars and AGNs, both of which are very distinctive on objective prism plates.

Boyle et al. (1995) are following a procedure similar to that of the EMSS (section 3.1). As many as $35 \%$ of the possible optical counterparts within 20 arcsec will be chance coincidences, with multiple candidates for some sources. In such cases, optical spectroscopy is carried out in order of increasing distance from the nominal source position until a plausible identification (e.g. QSO or $M$ star) can be claimed.

\section{Are Schmidt Plates accurate enough for 2dF Astrometry?}

One particular technique for identifying large numbers of sources is to use multiobject spectrographs fed by optical fibres. For example, with the new $2 \mathrm{dF}$ system soon to be completed on the AAT (Taylor, 1995), it will be possible to obtain 400 spectra simultaneously over a field two degrees in diameter. However, obtaining sufficiently accurate positions for candidate objects is not a trivial exercise. With fibres 2 arcsec in diameter, the positions must be accurate to about 0.2 arcsec r.m.s. While such accuracy has long been achievable on Schmidt plates (e.g. Anderson, 1971), is has often not been realised in more recent data. The challenge is to obtain positions accurate to this level over two degrees or more, and over a wide range of magnitudes from faint target objects to the relatively bright fiducial stars, needed for field acquisition and tracking. The biggest single source of error is probably the proper motions of the relatively bright reference stars; to achieve the necessary precision requires either a recent photographic plate or else a pair of plates so that proper motions can be allowed for. It is also necessary to worry about the 'magnitude equation'. Since the photographic plate is a non-linear detector with a limited dynamic range, any tracking or guiding errors are liable to show up as a systematic apparent shift in position of bright stars relative to faint stars, while additional effects arise from various circumstellar reflection ghost images, some of which are eccentric. These effects cannot be completely eliminated but they can be minimised, for example by measuring several plates and avoiding excessively bright guide stars. Fortunately it is not so important to get very accurate absolute positions, so long as guide stars and targets are all measured on the same system, since any zero-point error will be taken out in the telescope pointing.

\section{Conclusions}

- Experience in radio astronomy shows that many identifications can be made from relatively poor positions, once the main types of sources have been defined. However, such identifications will always be of limited reliability and completeness.

- Very accurate positions are required for finding new or rare classes of object. Although additional selection criteria can sometimes improve the 
efficiency of specific searches, e.g. for very high redshift objects, it is surprisingly difficult to make a dramatic improvement in the success rate, and such criteria can add an unquantifiable bias.

- The accuracy of optical positions is now the limiting factor in linking the radio and optical reference frames. More reference sources are also needed; a significant number of bright quasars must remain to be discovered in the south.

- For most other wavebands, e.g. X-ray and infrared, the accuracy of the source positions is still the limiting factor.

- Digitised optical databases can now be used for automatic identification of large samples of sources. However, careful verification is needed for individual sources, particularly if the claimed identification is with a rare or peculiar object.

- Plates from Schmidt Telescopes can provide high accuracy over wide fields, e.g. a precision of 0.2 arcsec over two degrees as required for multi-object spectroscopy with fibres, but only if suitable plates exist, if the field distortions are well understood, and if great care is taken.

\section{References}

Anderson J., 1971, A\&A, 13, 40

Blumenthal G. \& Miley G., 1979, A\&A, 80, 13

Bolton J. G., Clarke M. E. \& Ekers, R. D., 1965, Aust J Phys, 18, 627

Bolton J. G., Gardner F. F. \& Mackey M. B., 1964, Aust J Phys, 17, 340

Bolton J. G., Stanley J. G. \& Slee O. B., 1949, Nature, 164, 101

Bolton J. G., Wright A. E. \& Savage A., 1979, Aust J Phys Supp No. 46, p. 1

Boyle B. J., McMahon R. G., Wilkes B. J. \& Elvis M., 1995, MNRAS, 272, 462

Clowes R. G., Leggett S. K. \& Savage A., 1991, MNRAS, 250, 597

Fleming T. A., Green R. F., Jannuzi B. T., Liebert J., Smith P. S. \& Fink H., 1993, AJ, 106, 1729

Gioia I. M., Maccacaro T., Schild R., Wolter A., Stocke J. T., Morris S. L. \& Henry J. P., 1990, ApJ Supp, 72, 567

Green R. F., Schmidt M. \& Liebert J., 1986, ApJ Supp, 61, 305

Gregory P. C. \& Condon J. J., 1991, ApJ Supp, 75, 1011

Griffith M. R. \& Wright A. E., 1993, AJ, 105, 1666

Hazard C., Mackey M. B. \& Shimmins A.J., 1963, Nature, 197, 1037

Hewitt A. \& Burbidge G., 1987, ApJ Supp, 63, 1

Hunstead R.W., 1994, Aust J Phys, 47, 657

Irwin M. 1994. IAU Working Group on Wide-field Imaging, Newsletter No. 5 25

Jauncey D. L., 1991, Aust J Phys, 44, 785

Jauncey D. L., Batty M. J., Gulkis S. \& Savage, A., 1982, AJ, 87, 763 
Jauncey D. L., Savage A., Morabito D. D., Preston R. A., Nicolson G. D. \& Tzioumis A. K., 1989, AJ, 98, 54

Johnston K. J., 1994, in Very High Angular Resolution Imaging, IAU Symp. 158, eds J. G. Robertson \& W. J. Tango, Kluwer, p. 423

Kellermann K. I., Sramek R., Schmidt M., Shaffer D. B. \& Green R., 1989, ApJ, 98,195

McCarthy P. J., 1993, ARA\&A, 31, 639

Matthews T. A. \& Sandage A. R., 1963, ApJ, 138, 30

Moshir M., Kapan G., Conrow T., McCallon H., Hacking P., Gregorich D., Melnyk M., Rohrbach G., et al. 1989, Explanatory Supplement to the IRAS Faint Source Survey, JPL, Pasadena

Murdoch H.S., 1993, Proc ASA, 10, 346

O'Dea C. P., 1990, MNRAS, 245, 20P

Peterson B.A., Savage A., Jauncey D. L. \& Wright A. E., 1982, ApJ, 260, L27

Reynolds J. E. 1994, in Very High Angular Resolution Imaging, IAU Symp. 158, eds J. G. Robertson \& W. J. Tango, Kluwer, p. 437

Röser S. \& Bastian U., 1988., A\&A Supp, 74, 449

Sandage A. R., 1965, ApJ, 141, 1560

Savage A., Clowes R. G., MacGillivray H. T., Wolstencroft R. D., Leggett S. K. \& Puxley P. J. 1987, in Star Formation in Galaxies, NASA Conference Publ. 2466, ed. C. J. Lonsdale Persson, NASA, p. 537

Schmidt M., 1963, Nature, 197, 1040

Schmidt M. \& Green R. F., 1983, ApJ, 269, 352

Sealey K. M., Drinkwater M. J., Irwin M. J. \& Webb J. K., 1995, these proceedings

Staveley-Smith L., Manchester R. N., Kesteven M. J., Campbell-Wilson D., Crawford D. F., Turtle A. J., Reynolds J. E., Tzioumis A. K., Killeen N. E. B. \& Jauncey D. L., 1992, Nature, 355, 147

Stobie R. S., Kilkenny D., O'Donoghue, D. \& Chen A., 1995, these proceedings

Stocke J. T., Morris S. L., Gioia I. M., Maccacaro T., Schild R., Wolter A., Fleming T. A. \& Henry J. P., 1991, ApJ Supp, 76, 813

Sutherland W. J., McMahon R. G., Maddox S. J., Loveday J. \& Saunders W., 1991, MNRAS, 248, 483

Sutherland W. \& Saunders W., 1992, MNRAS, 259, 413

Taff L. G., Lattanzi M. G. \& Bucciarelli B., 1990, ApJ, 358, 359

Tasker N. J., Wright A. E., McConnell D. J., Savage A., Kesteven M. J., Troup E. \& Griffith M., 1993, Proc ASA, 10, 320

Taylor K., 1995, in Proceedings of the 35th Herstmonceux Conference, Cambridge, in press

Unewisse A. M., Hunstead R.W. \& Piestrzynski B., 1993, Proc ASA, 10, 229

Voges W., 1992, Astrophys. Sp. Sci. Ser., 174, 453; proceedings of Digitised Optical Sky Surveys, eds H. T. MacGillivray \& E. B. Thomson

White G. L., Jauncey D. L., Reynolds J. E., Blackmore D. R., Matcher S. J., Morgan B. L., Vine H. A. \& Argue A. N., 1991, MNRAS, 248, 411 
Wolstencroft R. D., Savage A., Clowes R. G., MacGillivray H. T., Leggett S. K. \& Kalafi M., 1986, MNRAS, 223, 279

Wolstencroft R. D., MacGillivray H. T., Lonsdale C. J., Conrow T., Yentis D. J., Wallin J. F. \& Hau G., 1992, Astrophys. Sp. Sci. Ser., 174, 471; proceedings of Digitised Optical Sky Surveys, eds H. T. MacGillivray \& E. B. Thomson

Yentis D. J., Cruddace R. G., Gursky H., Stuart B. V., Wallin J. F., MacGillivray H. T. \& Collins C. A., 1992, Astrophys. Sp. Sci. Ser., 174, 67; proceedings of Digitised Optical Sky Surveys, eds H. T. MacGillivray \& E. B. Thomson

\section{Discussion}

Hawkins: A comment - I agree that there is a problem of accuracy at the one arcsec level which I believe is due to COSMOS. There is a magnitude effect between the bright astrometric standards and faint programme stars.

Collins: I wonder whether we are nearing the point at which Schmidt plates will be useful for optical identifications. For example, a substantial fraction of the sources in the ROSAT all-sky survey are clusters and AGN's below the plate limit. Surely, this is a further argument for digitised surveys.

Cannon: That is true for certain classes of objects. It depends what fraction of the sources fall below the plate limit; there have always been some 'blank field' objects in catalogues of radio sources, for example, but the Schmidt surveys are still a very useful starting point. 